Received: 10 November 2009, Accepted: 26 November 2009 Edited by: A. Goñi

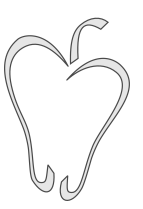

ISSN 1852-4249

\title{
Light effect in photoionization of traps in GaN MESFETs
}

\author{
H. Arabshahi, ${ }^{1 *}$ A. Binesh ${ }^{2}$
}

\begin{abstract}
Trapping of hot electron behavior by trap centers located in buffer layer of a wurtzite phase GaN MESFET has been simulated using an ensemble Monte Carlo simulation. The results of the simulation show that the trap centers are responsible for current collapse in GaN MESFET at low temperatures. These electrical traps degrade the performance of the device at low temperature. On the opposite, a light-induced increase in the traplimited drain current, results from the photoionization of trapped carriers and their return to the channel under the influence of the built in electric field associated with the trapped charge distribution. The simulated device geometries and doping are matched to the nominal parameters described for the experimental structures as closely as possible, and the predicted drain current and other electrical characteristics for the simulated device including trapping center effects show close agreement with the available experimental data.
\end{abstract}

\section{Introduction}

GaN has become an attractive material for power transistors [1-3] due to its wide band gap, high breakdown electric field strength, and high thermal conductivity. It also has a relatively high electron saturation drift velocity and low relative permitivity, implying potential for high frequency performance. However, set against the virtues of the material are the disadvantages associated with material quality. GaN substrates are not readily available and the lattice mismatch of GaN to the different substrate materials commonly used means that layers typically contain between $10^{8}$ and $10^{10}$ threading dislocations per $\mathrm{cm}^{2}$. Further, several types of electron traps occur in the device

\footnotetext{
*E-mail: arabshahi@um.ac.ir

1 Department of Physics, Ferdowsi University of Mashhad, P.O. Box 91775-1436, Mashhad, Iran.

2 Department of Physics, Payam-e-Nour University, Fariman, Iran.
}

layers and have a significant effect on $\mathrm{GaN}$ devices. In the search for greater power and speed performance, the consideration of different aspects that severely limit the output power of GaN FETs must be accounted for. It is found that presence of trapping centers in the GaN material is the most important phenomenon which can effect on current collapse in output drain current of GaN MESFET. This effect was recently experimentally investigated in GaN MESFET and was observed that the excess charge associated with the trapped electrons produces a depletion region in the conducting channel which results in a severe reduction in drain current [4]. The effect can be reversed by librating trapped electrons either thermally by emission at elevated temperatures or optically by photoionization. There have been several experimental studies of the effect of trapping levels on current collapse in GaN MESFET. For example, Klein et al. [5-6] measured photoionization spectroscopy of traps in GaN MESFET transistors and calculated that the current collapse resulted from charge trapping in the buffer layer. Binari et 
PAPers in Physics, vol. 1, ART. 010006 (2009) / H. Arabshahi et al.

al. [7] observed decreases in the drain current of a GaN FET corresponding to the deep trap centers located at 1.8 and $2.85 \mathrm{eV}$.

In this work, we report a Monte Carlo simulation which is used to model electron transport in wurtzite GaN MESFET including a trapping centers effect. This model is based upon the fact that since optical effect can emit the trapped electrons that are responsible for current collapse, the incident light wavelength dependence of this effect should reflect the influence of trap centers on hot electron transport properties in this device. This article is organized as follows. Details of the device fabrication and trapping model which is used in the simulated device are presented in section 2, and the results from the simulation carried out on the device are interpreted in section 3.

\section{Model, device and simulations}

An ensemble Monte Carlo simulation has been carried out to simulate the electron transport properties in GaN MESFET. The method simulates the motion of charge carriers through the device by following the progress of $10^{4}$ superparticles. These particles are propagated classically between collisions according to their velocity, effective mass and the prevailing field. The selection of the propagation time, scattering mechanism and other related quantities, is achieved by generating random numbers and using these numbers to select, for example, a scattering mechanism. Our self-consistent Monte Carlo simulation was performed using an analytical band structure model consisting of five nonparabolic ellipsoidal valleys. The scattering mechanisms considered for the model are acoustic and polar optical phonon, ionized impurity, piezoelectric and nonequivalent intervalley scattering. The nonequivalent intervalley scattering is between the $\Gamma_{1}, \Gamma_{3}, \mathrm{U}, \mathrm{M}$ and $\mathrm{K}$ points.

The parameters used for the present Monte Carlo simulations for wurtzite $\mathrm{GaN}$ are the same as those used by Arabshahi for MESFET transistors [8-9]. The device structure illustrated in figure 1.a is used in all simulations. The overall device length is 3.3 $\mu \mathrm{m}$ in the $x$-direction and the device has a $0.3 \mu \mathrm{m}$ gate length and $0.5 \mu \mathrm{m}$ source and drain length.

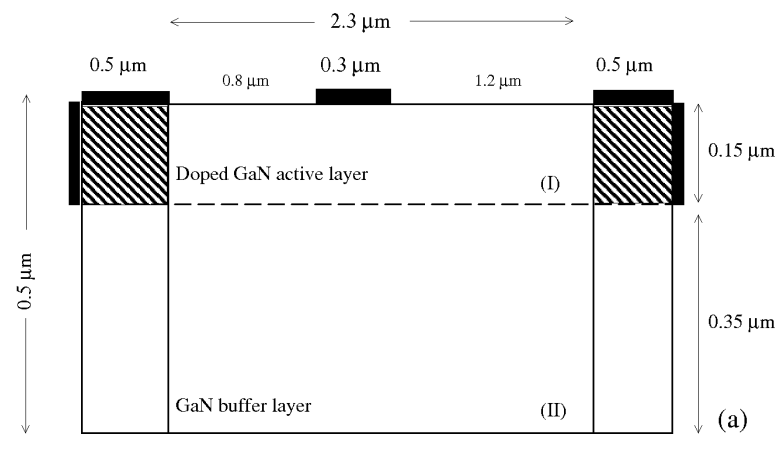

$$
\begin{array}{lll}
\text { N } & 5^{*} 10^{23} \mathrm{~m}^{-3} & \\
\text { (I) } 2 * 10^{23} \mathrm{~m}^{-3} & \text { Source \& Drain = Ohmic Contacts } \\
\text { (II) } 1^{*} 10^{22} \mathrm{~m}^{-3} & \text { Gate } \quad=\text { Schottky Contact }
\end{array}
$$

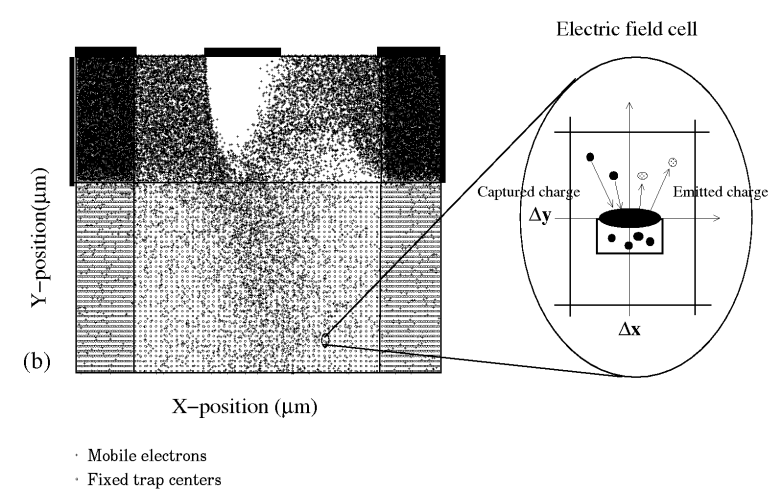

Figure 1: (a) Cross section of wurtzite GaN MESFET structure which we have chosen in our simulation. Source and drain contacts have low resistance ohmic contacts, while the gate contact forms a Schottky barrier between the metal and the semiconductor epilayer, (b) The instantaneous distribution of $10^{4}$ particles at steady forward bias (drain voltage $50 \mathrm{~V}$, gate voltage $-1 \mathrm{~V}$ ), superimposed on the mesh. Note that in the simulation there are two types of superparticles. The mobile particles which describe unbound electron flow through the device and trapping center particles which are fixed at the center of each electric field cell (in this case in the buffer layer only). The ellipse represents a trap center which is fixed at the center of an electric field cell and occupied by some mobile charges.

The source and drain have ohmic contacts and the gate is in Shottky contact in $1 \mathrm{eV}$ to reperesent the contact potential at the $\mathrm{Au} / \mathrm{Pt}$. The source and drain regions are doped to $5 \times 10^{23} \mathrm{~m}^{-3}$ and the 
Papers in Physics, vol. 1, ART. 010006 (2009) / H. Arabshahi et al.

top and down buffer layers are doped to $2 \times 10^{23}$ $\mathrm{m}^{-3}$ and $1 \times 10^{22} \mathrm{~m}^{-3}$, respectively. The effective source to gate and gate to drain separation are 0.8 $\mu \mathrm{m}$ and $1.2 \mu \mathrm{m}$, respectively. The large dimensions of the device need a long simulation time to ensure convergence of the simulator. The device is simulated at room temperature and $420 \mathrm{~K}$.

In the interests of simplicity it is assumed that there is just a single trap with associated energy level $E_{T}$ in all or just part of the device. Further, it is assumed that only electrons may be captured from the conduction band by the trap centers, which have a capture cross-section $\sigma_{n}$ and are neutral when unoccupied, and may only be emitted from an occupied center to the conduction band. We use the standard model of carrier trapping and emission [9-10].

For including trapping center effects, the following assumption has been considered. The superparticles in the ensemble Monte Carlo simulation are assumed to be of two types. There are mobile particles that represent unbound electrons throughout the device. However, the particles may also undergo spontaneous capture by the trap centers distributed in the device. The other type of superparticles are trapping centers that are fixed at the center of each mesh cell. As illustrated in figure 1.b, each trap center has the capacity to trap a finite amount of mobile electronic charge from particles that are in its vicinity and reside in the lowest conduction band valley. The vicinity is defined as exactly the area covered by the electric field mesh cell. The finite capacity of the trapping center in each cell of a specific region in the device is set by a density parameter in the simulation programme. The simulation itself is carried out by the following sequence of events. First, the device is initialized with a specific trap which is characterized by its density as a function of position, a trap energy level and a capture cross-section. Then at a specific gate bias the source-drain voltage is applied.

Some of the mobile charges passing from the source to the drain in each timestep can be trapped by the centers with a probability which is dependent on the trap cross-section and particle velocity in the cell occupied at the relevant time $t$. The quantity of charge that is captured from a passing mobile particle is the product of this probability and the charge on it. This charge is deducted from the charge of the mobile particle and added to the fixed charge of the trap center. The emission of charge is simulated using the emission probability. Any charge emitted from a trap center is evenly distributed to all mobile particles in the same field cell. Such capture and emission simulations are performed for the entire mesh in the device and information on the ensemble of particles is recorded in the usual way.

\section{Results}

The application of a high drain-source voltage causes hot electrons to be injected into the buffer layer where they are trapped by trap centers. The trapped electrons produce a depletion region in the channel of the GaN MESFET which tends to pinch off the device and reduce the drain current. This effect can be reversed by any factor which substantially increases the electron emission rate from the trapped centers, such as the elevated temperatures considered previously. Here we consider the effect of exposure to light [11-13].

There have been several experimental investigations of the influence of light on the device characteristics. Binari et al. [6] were the first to experimentally study the current collapse in GaN MESFETs as a function of temperature and illumination. They showed that the photoionization of trapped electrons in the high-resistivity GaN layers and the subsequent return of these electrons to the conduction band could reverse the drain current collapse. Their measurements were carried out as a function of incident light wavelength with values in the range of $380 \mathrm{~nm}$ to $720 \mathrm{~nm}$, corresponding to photon energies up to $3.25 \mathrm{eV}$ which is close to the GaN band gap. Their results show that when the photon energy exceeds the trap energy, the electrons are quickly emitted and a normal set of drain characteristics is observed.

To examine the photoionization effect in our simulations, the thermal emission rate $e_{n}^{t}$ was changed to $e_{n}^{t}+e_{n}^{o}$, where $e_{n}^{o} \sim \sigma_{n}^{o} \Phi$ is the optical emission rate, with $\sigma_{n}^{o}$, the optical capture cross-section and $\Phi$ the photon flux density given by

$$
\Phi=\frac{I}{h \nu}=\frac{I \lambda}{h c}
$$

where $I$ is the light intensity, $\nu$ is the radiation frequency and $\lambda$ is the incident light wavelength.

Our modeling of photoionization effects in GaN 
PAPers in Physics, vol. 1, ART. 010006 (2009) / H. Arabshahi et al.
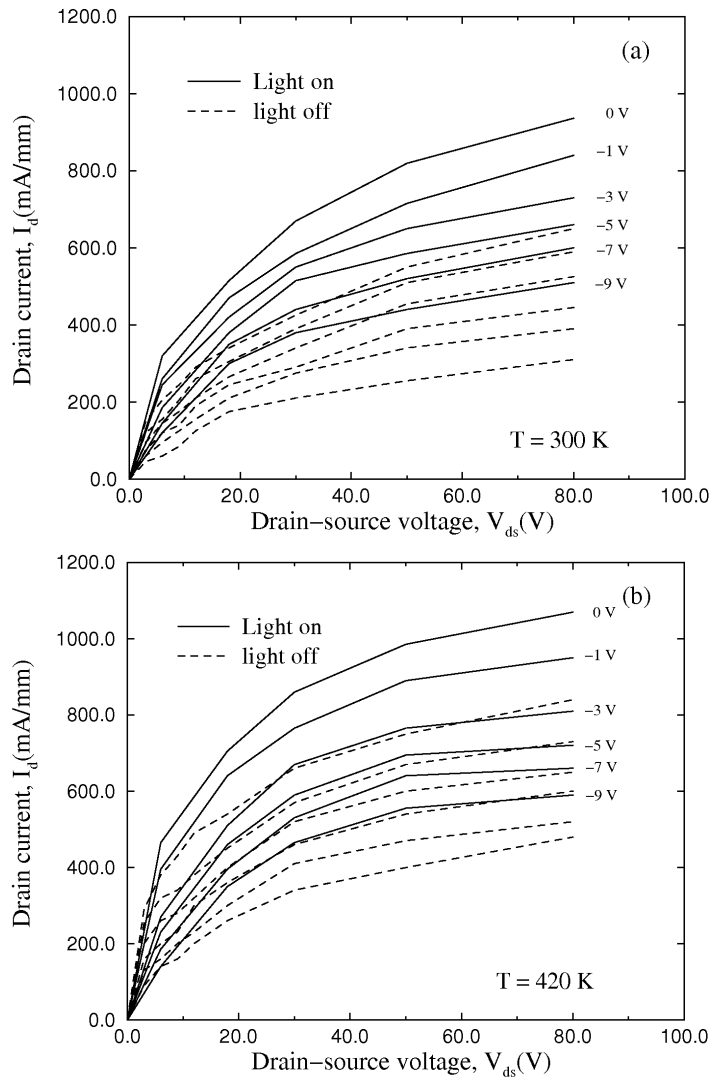

Figure 2: $I-V$ characteristics of a GaN MESFET under optical and thermal emission of trapped electrons (solid curve) and thermal emission of trapped electrons (dashed curve) at two different temperatures. (a) At $T=300 \mathrm{~K}$ with trap centers at 1.8 $\mathrm{eV}$ and illuminated with a photon energy of 2.07 eV. (b) At $T=420 \mathrm{~K}$ with trap centers at $2.85 \mathrm{eV}$ and illuminated with a photon energy of $3.1 \mathrm{eV}$.

MESFETs is based on parameters used by Binari and Klein [5-7]. The simulations were all carried out for two different deep trap centers, both with a concentration of $10^{22} \mathrm{~m}^{-3}$, and with photoionization threshold energies at 1.8 and $2.85 \mathrm{eV}$ and capture cross-sections of $6 \times 10^{-21} \mathrm{~m}^{2}$ and $2.8 \times 10^{-19}$ $\mathrm{m}^{2}$, respectively. A fixed incident light intensity of $5 \mathrm{Wm}^{-2}$ at photon energies of $2.07 \mathrm{eV}$ and $3.1 \mathrm{eV}$ is used. The simulations have been performed at a sufficiently high temperature $(420 \mathrm{~K})$, for both thermal and optical emission, to be significant as well as at room temperature.

Figure 2a illustrates the effect on the drain current characteristics of exposure of the device to light at room temperature. The GaN MESFET has a deep trap center at $1.8 \mathrm{eV}$ and is illuminated at a photon energy of $2.07 \mathrm{eV}$. It can be seen that in the light the $I$ - $V$ curves generally exhibit a larger drain current, especially at higher drain voltages, reflecting the fact that the density of trapped electrons is much lower.

Simulations have also been performed at $420 \mathrm{~K}$ for a device with deep level traps at $2.85 \mathrm{eV}$. The simulation results in figure $2 \mathrm{~b}$ for illumination of a photon energy of $3.1 \mathrm{eV}$ are compared with the collapsed $I$ - $V$ curves in the absence of light. Comparison of figures $2 \mathrm{a}$ and $2 \mathrm{~b}$ shows that the currents are generally higher at $420 \mathrm{~K}$ and that the light has less effect at the highest temperature.

\section{Conclusions}

The dependence upon light intensity (exposure) of the reversal of current collapse was simulated in a GaN MESFET for a single tapping center. Traps in the simulated device produce a serious reduction in the drain current and consequently the output power of GaN MESFET. The drain current behavior as a function of illumination with photon energy was also studied. Our results show that as the temperature and photon energy are increased, the collapsed drain current curve moves up toward the non-collapsed curve due to more emission of trapped electrons.

Acknowledgements - The authors wish to thank M. G. Paeezi for the helpful comments and critical reading of the manuscript.

[1] B Gil, Group-III Nitride Semiconductor Compounds, Oxford Science Pub. (1998).

[2] M A Khan, M S Shur, AlGaN/GaN Metal Oxide Semiconductor Heterostructure Field Effect Transistor, Mater. Sci. Eng. B 42, 69 (1997).

[3] P B Klein, S C Binari, J A Freitas, A E Wickenden, Photoionization spectroscopy of traps in GaN metal-semiconductor field-effect transistors, J. Appl. Phys. 88, 2843 (2000). 
[4] M A Khan, M S Shur, Q C Chen, J N Kuznia, Low frequency noise in GaN metal semiconductor and metal oxide semiconductor field effect transistors, Electron. Lett. 30, 2175 (1994).

[5] P B Klein, S C Binari, J A Freitas, A E Wickenden, Observation of deep traps responsible for current collapse in GaN metalsemiconductor field-effect transistors, J. Appl. Phys. 88, 2843 (2000).

[6] P B Klein, J A Freitas, S C Binari, A E Wickenden, AlGaN/GaN heterostructure fieldeffect transistor model including thermal effects, Appl. Phys. Lett. 75, 4016 (1999).

[7] S C Binari, W Kruppa, H B Dietrich, G Kelner, A E Wickenden, J A Freitas, Trapping effects and microwave power performance in AlGaN/GaN HEMTs, Solid State Electron. 41, 1549 (1997).

[8] H Arabshahi, Monte Carlo simulations of electron transport in Wurtzite phase GaN MESFET including trapping effect, Modern Phys. Lett. B 20, 787 (2006).
[9] H Arabshahi, The frequency response and effect of trap parameters on the characteristic of GaN MESFETs, The Journal of Damghan University of Basic Sciences 1, 45 (2007).

[10] S Trassaert, B Boudart, C Gaquiere, Investigation of traps induced current collapse in GaN devices, a1404 ORSAY France, 127 (1999).

[11] A Kastalsky, S Luryi, A C Gossard, W K Chan, Switching in NERFET circuits, IEEE Electron Device Lett. 6, 347 (1985).

[12] J C Inkson, Deep impurities in semiconductors. II. The optical cross section, J. Phys. C: Solid State Phys. 14, 1093 (1981).

[13] D V Lang, R A Logan, M Jaros, Monte Carlo evaluations of degeneracy and interface roughness effects, Phys. Rev. B 19, 1015 (1979). 